\title{
Regulation of KIR3DL3 Expression via miRNA
}

\author{
Rungtiwa Nutalai ${ }^{1,2,3}$, Silvana Gaudieri ${ }^{4,5,6}$ (D) Amonrat Jumnainsong ${ }^{2,3}$ and \\ Chanvit Leelayuwat $2,3, *$ (D) \\ 1 Biomedical Sciences Program, Graduates School of Khon Kaen University, Khon Kaen 40002, Thailand \\ 2 Department of Clinical Immunology and Transfusion Sciences, School of Medical Technology, Faculty of \\ Associated Medical Sciences, Khon Kaen University, Khon Kaen 40002, Thailand \\ 3 The Centre for Research and Development of Medical Diagnostic Laboratories (CMDL), Faculty of \\ Associated Medical Sciences, Khon Kaen University, Khon Kaen 40002, Thailand \\ 4 School of Human Sciences, University of Western Australia, Perth, WA 6009, Australia \\ 5 Institute for Immunology and Infectious Diseases, Murdoch University, Perth, WA 6150, Australia \\ 6 Division of Infectious Diseases, Vanderbilt University Medical Center, Nashville, TN 37232, USA \\ * Correspondence: chanvit@kku.ac.th; Tel.: +66-813-923802
}

Received: 16 July 2019; Accepted: 5 August 2019; Published: 9 August 2019

\begin{abstract}
Killer-cell immunoglobulin-like receptor (KIR) 3DL3 is a framework gene present in all human KIR haplotypes. Although the structure of KIR3DL3 is suggestive of an inhibitory receptor, the function of KIR3DL3 has not been demonstrated and cognate ligands have not been identified. KIR3DL3 has been shown to be constitutively expressed at a low RNA level in peripheral blood mononuclear cell (PBMC) and decidual natural kill (NK) cells, but cell surface expression of KIR3DL3 cannot be detected. Accordingly, post-transcriptional regulation of KIR3DL3 should exist. Using bioinformatics analysis, we identified three candidate micro ribonucleic acids (miRNAs; miR-26a-5p, -26b-5p and -185-5p) that potentially regulate KIR3DL3 expression. Luciferase reporter assays utilizing constructs with mutated miRNA-binding sites of miR-26a-5p, -26b-5p and -185-5p in the 3'-untranslated region (3' UTR) of KIR3DL3 resulted in up-regulation of luciferase activity demonstrating a potential mechanism of gene regulation. Furthermore, knockdown of the same endogenous miRNAs using silencing ribonucleic acid (siRNA) led to induced surface expression of KIR3DL3. In conclusion, we provide a novel mechanism of functional regulation of KIR3DL3 via miRNAs. These findings are relevant in understanding the generation of KIR repertoire and NK cell clonality.
\end{abstract}

Keywords: killer-cell immunoglobulin-like receptor (KIR) 3DL3; miRNA; 3'untranslated region; post-transcriptional regulation

\section{Introduction}

Killer-cell immunoglobulin-like receptors (KIRs) are transmembrane glycoproteins consisting of both inhibitory and activating receptors on natural kill (NK) cells and on a subset of T cells [1,2]. NK cells exhibit at least one inhibitory receptor to discriminate cells with aberrant human leucocyte antigen (HLA) class I surface expression often observed during infection and malignant transformation from normal cells [3]. This receptor-ligand interaction is important in establishing the NK cell activation threshold and is influenced by the diversity in both sets of molecules. KIR diversity is generated through: (I) Allelic variations, (II) the level of surface expression, and (III) haplotypic diversity by the presence or absence of KIR genes. Furthermore, each individual NK cell can express different KIR repertoires ranging from one to eight receptors to generate NK clonality $[4,5]$. For example, four conserved KIR genes are present in virtually all individuals, known as the framework genes KIR3DL3, KIR3DP1, KIR2DL4, and KIR3DL2 [6,7], but only KIR2DL4 is expressed at both the RNA and protein 
level in all individuals $[4,8]$. It is still unclear how different KIR repertoires are generated from the same genetic template but the distribution of KIRs appears to be partly determined by DNA methylation releasing a broad range of functional responses [9-11].

KIR3DL3, also known as KIRC1, KIR44, and KIR3DL7, is a member of the inhibitory KIR surface receptors possessing three extracellular immunoglobin (Ig) domains (3D) and only one inhibitory motif within the long cytoplasmic domain (L) due to a premature stop codon [12-14]. Moreover, exon 6 of KIR3DL3 encoding the stem part of the receptor is also absent, which differs from other inhibitory KIRs. Understanding the function of KIR3DL3 is further complicated by the unknown identity of the specific ligand, but its importance is highlighted by its presence in all human KIR haplotypes with 120 distinct polymorphic alleles of the coding sequence [12,15].

An unusual feature of KIR3DL3 is its limited detection at the mRNA level as reflected by its methylation status [12,16,17], even though the KIR3DL3 promoter shows the strongest activity of all KIR genes [17]. However, KIR3DL3 mRNA expression has been recently identified in decidual NK cells and in the cluster of differentiation (CD) $56^{\text {bright }} \mathrm{NK}$ cell subset in peripheral blood mononuclear cells (PBMCs) with females showing higher levels of expression than males, but there is a lack of KIR3DL3 protein expression [16]. Accordingly, it has been proposed that KIR3DL3 is expressed in specific pathological situations or during development. However, regulation of KIR3DL3 mRNA at the post-transcriptional level remains elusive.

Microribonucleic acids (miRNAs) are small non-coding RNA regulators of approximately 21 nucleotides that act at a post-transcriptional step of gene expression affecting diverse fundamental biological processes including cellular immune functions and development [18-20]. These non-coding RNAs inhibit protein synthesis by binding to the 3'-untranslated region (3'-UTR) of target mRNAs in a partially complementary fashion [21,22]. In silico prediction analysis shows that many miRNAs can interact with KIR3DL3, therefore the aim of the current study was to identify the miRNAs that downregulate KIR3DL3.

Here, we provide evidence that the miRNAs miR-26a-5p, miR-26b-5p, and miR-185-5p control KIR3DL3 expression. This pathway may be genetically manipulated to increase broad cytotoxicity of NK cells through potential target miRNAs.

\section{Materials and Methods}

\subsection{Cell Culture}

Three NK cell lines NK92, YT, and YT-KIR3DL3 and the K562 cell line were used in this study. YT-KIR3DL3 cells were developed by stable transfection of YT cells with a plasmid containing full-length KIR3DL3. These cells can constitutively express the KIR3DL3 protein on the surface [16]. YT-KIR3DL3 cells were kindly provided by Professor Ashley Moffet (University of Cambridge, Cambridge, UK). Cells were maintained in the medium Roswell Park Memorial Institute (RPMI) 1640 supplemented with $10 \%$ heat-inactivated fetal bovine serum, $2 \mathrm{mM} \mathrm{L}$-glutamine, $100 \mathrm{U} / \mathrm{mL}$ penicillin, $100 \mu \mathrm{g} / \mathrm{mL}$ streptomycin (Gibco, Grand Island, NY, USA), $0.1 \mathrm{mM}$ 2-mercaptoethanol and $100 \mathrm{U} / \mathrm{mL}$ recombinant human interleukin-2 (IL-2; Sigma, St. Louis, MO, USA) for IL-2-dependent NK92 cells. All cells were cultured at $37{ }^{\circ} \mathrm{C}$ with $5 \% \mathrm{CO}_{2}$.

\subsection{Bioinformatic Prediction of Potential miRNAs for KIR3DL3}

All 2,588 reported human miRNA data were obtained from miRBase (http://www.mirbase.org/) [23], and all allelic 3'-UTR sequences of KIR3DL3 were retrieved from the IPD-KIR Database (https: //www.ebi.ac.uk/ipd/kir). To predict the potential miRNA that may regulate KIR3DL3 by acting on the 3'UTR of the gene, we utilized multiple prediction programs with inherently different algorithms, including RNAhybrid [24], Targetscan [25] and miRanda [26]. The candidate miRNAs were selected from three integrative searches. 


\subsection{Quantitative Real-Time Reverse Transcription Polymerase Chain Reaction (qRT-PCR)}

Total RNAs, including miRNAs and mRNAs, were extracted using the Illustra RNAspin Mini RNA Isolation Kit (GE Healthcare Life Sciences, Buckinghamshire, Buckingham, UK), according to the manufacturer's protocol. The RNAs were converted to cDNAs using the Moloney murine leukemia virus reverse transcriptase (M-MLV RT) (Promega, Madison, MD, USA) and random hexamers. In addition, reverse-transcription of mature miRNAs was performed using stem-loop reverse transcript primers [27,28].

Amplification of cDNA was performed using specific primers [29] (Supplementary Table S1) and the QuantiFast SYBR Green PCR kit (QIAGEN, Germantown, MD, USA) on an ABI PRISM 7500 Real-Time PCR system (Applied Biosystems, Foster City, MA, USA). RNA polymerase II (RPII) was used as an endogenous control for KIR3DL3 and miR-16 for miRNA. Relative gene expression was calculated as $2^{\Delta C T}: \Delta C_{T}=C_{T}$ KIR3DL3 - $C_{T}$ RPII. For miRNA detection, amplification was performed using specific forward primers and universal reverse primer [28] and relative expression calculated as $2^{\Delta \mathrm{CT}}: \Delta \mathrm{C}_{\mathrm{T}}=\mathrm{C}_{\mathrm{T}}$ miRNA $-\mathrm{C}_{\mathrm{T}}$ miR-16.

\subsection{Reporter Plasmid Construction and Luciferase Reporter Assay}

Luciferase vectors, firefly and renilla plasmids (pcDNA3.1-Zeo(+)Pp and pRL-SV40, respectively), were kindly provided by Dr Yong Sun Lee, University of Texas Medical Branch, USA. Specific primers were used for the amplification of wild-type 3'UTR KIR3DL3 (p3UTR_WT) and site-specific miRNA mutagenesis (pMu_miR) (primer sequences in Supplementary Table S1). All fragments were amplified from the genomic DNA of NK92 and subsequently inserted between BamHI and XhoI downstream sites of the firefly gene in pcDNA3.1-Zeo(+)Pp, leading to the modified constructs as shown in Table 1. The sequences of the constructs were confirmed by Sanger-based DNA sequencing (Macrogen Inc., Seoul, Korea).

Table 1. List of plasmid constructs.

\begin{tabular}{cc}
\hline Plasmid Names & Information \\
\hline p3'UTR_WT & Wild type 3'UTR of KIR3DL3 \\
pMu_miR-26a/b-5p (295) & Mutated miR-26a/b-5p binding site 3'UTR at positions 297 and 299 \\
pMu_miR-26a/b-5p (344) & Mutated miR-26a/b-5p binding site 3'UTR at positions 344 and 348 \\
pMu_miR-26a/b-5p (295,344) & Mutated both miR-26a-5p and miR-26b-5p binding sites \\
pMu_miR-185-5p & Mutated miR-185-5p binding site \\
pMu_miR-203a-3p & Mutated miR-203a-3p binding site \\
\hline
\end{tabular}

In 24-well plates, $2.5 \times 10^{5} \mathrm{YT}$ cells were transiently co-transfected with $1.8 \mu \mathrm{g}$ of the pcDNA3.1-Zeo(+)Pp reporter plasmid or modified pcDNA3.1-Zeo(+)Pp containing the 3'UTR of KIR3DL3, and $0.2 \mu \mathrm{g}$ of the pRL-SV40 control vector encoding renilla luciferase (Promega, Madison, MD, USA) by using the FuGene ${ }^{\circledR}$ HD transfection reagent (Roche, Indianapolis, MA, USA). After $48 \mathrm{~h}$ of transfection, luciferase activities of each construct were measured by the Dual-luciferase reporter assay (Promega, Madison, MD, USA) by the GloMax ${ }^{\circledR}$ 20/20 luminometer machine (Promega, Madison, MD, USA). The firefly luciferase activity was normalized with the renilla luciferase activity and then normalized to the average activity of the control reporter. Experiments were performed in triplicates. The Paired Student's $t$-test was used to compare the average luciferase activities in various reporter vectors [30]. 


\subsection{Targeted miRNA Inhibition}

The mirVana®miRNA inhibitors for miR-26a-5p, miR-26b-5p, and miR-185-5p (Ambion, Austin, TX, USA) were used in this study with a negative control or scramble control. K562 cells were transfected with $500 \mathrm{nM}$ of inhibitors using the Neon®Transfection System (Invitrogen, Thermo Fisher Scientific Inc., Grand Island, NY, USA). The cells were then collected at $48 \mathrm{~h}$ after transfection and analyzed for the expression of mRNA and cell surface KIR3DL3 expression by flow cytometry.

\subsection{Flow Cytometry}

Cells were stained with mouse $\operatorname{IgG}_{2 B}$ isotype control (R\&D Systems, Minneapolis, MI, USA) or primary mouse anti-KIR3DL3 antibody kindly provided by Professor Ashley Moffet, University of Cambridge, UK [16]. Where necessary, a goat anti-mouse PE secondary antibody was used (R\&D Systems, Minneapolis, MI, USA). The level of KIR3DL3 expression was analyzed by the Beckman Coulter Gallios and the Kaluza Flow Analysis Software (Beckman Coulter, Fullerton, CA, USA). Percentage of positive cells and mean fluorescence intensity (MFI) were calculated following consideration of the values from the isotype control.

\subsection{Statistical Analysis}

All data were presented as mean \pm standard error of the mean of three independent experiments. All data were tested for normal distribution by the Shapiro-Wilk Test. The statistical differences between two groups were analyzed using the Student's $t$-test by GraphPadPro Prism5.0 (GraphPad, San Diego, CA, USA). A value of $p<0.05$ was considered a statistically significant difference, and the degree indicated as follows: ${ }^{*}, p<0.05 ;{ }^{* *}, p<0.01 ;{ }^{* * *}, p<0.001$.

\section{Results}

\subsection{Bioinformatic Prediction of Potential miRNA Candidates for KIR3DL3}

Initially, KIR3DL3 expression was evaluated in the NK92 cell line to confirm regulation at the post-transcriptional level. NK92 cells had detectable mRNA for KIR2DL4, 3DL1, and 3DL3 but lacked cell surface expression of KIR3DL3 (Table S2), supporting a post-transcription regulation mechanism.

Next, to identify the potential miRNAs targeting the 3'UTR of KIR3DL3, we used three bioinformatic programs RNAhybrid [24], TargetScan [25] and miRanda [26] to produce a target prediction list. Of the list of putative miRNA targets, 26 miRNAs were identified by all three programs (Figure 1a). In agreement with previous reports [31], six miRNAs were matched with the list of miRNAs expressed in human $\left(\mathrm{CD}^{2} 6^{+} \mathrm{CD}^{-}\right)$NK cells, namely miR-26a-5p, -26b-5p, -185-5p, -203a-3p, -372 and miR-373. To determine whether these miRNAs could contribute to the regulation of KIR3DL3 at the post-transcriptional step, NK92 and YT cells were analyzed for the expression of the six candidate miRNAs by Stem-loop qRT-PCR. Specifically, miR-26a-5p, -26b-5p and miR-185-5p were expressed in both NK cell lines, however miR-203a-3p was only expressed in NK92 cells, albeit at a low level (Figure $1 \mathrm{~b}$ and Supplementary Figure S1). Therefore, the three miRNAs expressed in all cell lines were selected as potential miRNA candidates regulating KIR3DL3. 


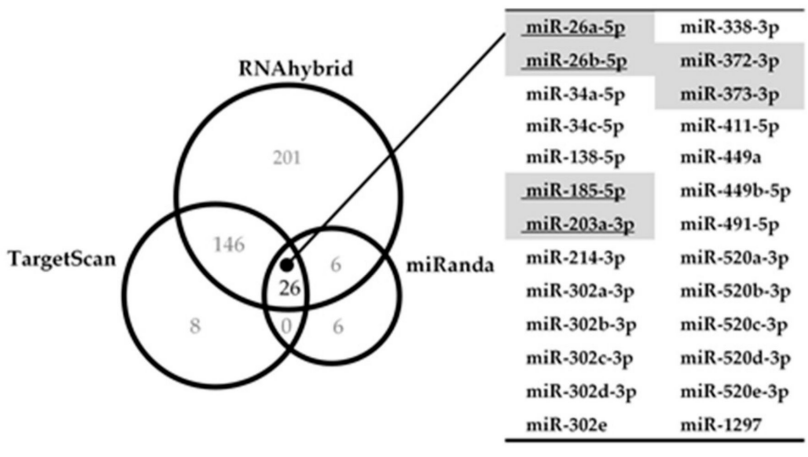

(a)

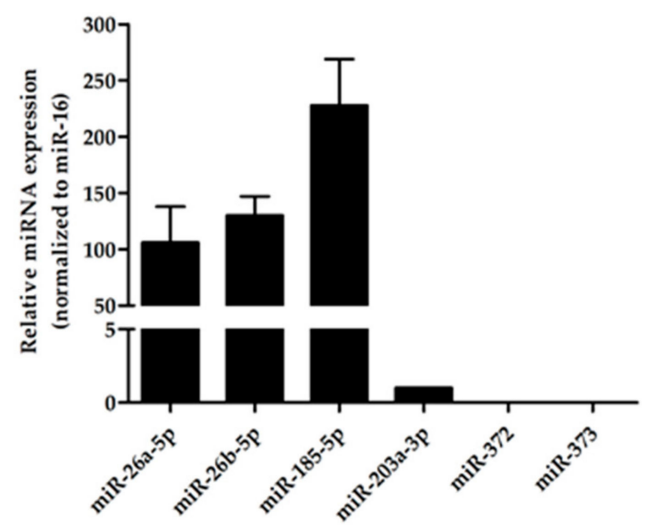

(b)

Figure 1. A summary of candidate miRNA prediction targets in KIR3DL3 and the endogenous expression of specific miRNAs. (a) Venn diagram representing the number of potential miRNAs for KIR3DL3 and the common miRNAs $(n=26)$ predicted from three different miRNA-target prediction software (RNAhybrid, TargetScan and miRanda). The table highlights six miRNAs matched with previously identified miRNAs in human $\left(\mathrm{CD} 6^{+} \mathrm{CD}^{-}\right) \mathrm{NK}$ cells [31]. Only four miRNA candidates were examined in the NK-92 cell line (underlined). (b) Endogenous expression of six miRNAs in NK-92 cells was determined by quantitative real-time polymerase chain reaction (qRT-PCR). The expression levels were normalized to miR-16. The results are depicted as mean \pm standard error of mean (S.E.M).

\subsection{Potential miRNA Candidates Targeting KIR3DL3 Through the 3'UTR}

To investigate the role of specific miRNAs binding to the 3'UTR of KIR3DL3, we constructed a panel of reporter plasmids containing wild-type and mutated miRNA binding sites of the KIR3DL3 3'UTR (Table 1). Site-directed mutagenesis was used to produce one plasmid containing a mutated site for each miRNA, and a second encompassing combinations of mutations ( $>2$ sites) to investigate synergistic effects of the miRNAs. For the miRNAs miR-26a-5p and 26b-5p they were identical at the seed sequences and had two target sites on the 3'UTR. The miRNA miR-203a-3p was present at a very low level in NK92 cells or absent in YT cells (as described above) and accordingly the mutation plasmid for the miR-203a-3p binding site (pMu_miR-203a-3p) acted as a control such that in the case of low level or absent expression of the miRNA, mutating the miRNA binding site should have no effect. These plasmid constructs were transfected into YT cells. As shown in Figure 2, luciferase activity was significantly decreased to $60 \%$ for the plasmid with the wild type $3^{\prime}$ UTR (p3UTR_WT) compared to the control (empty) vector. Moreover, point mutations at both binding sites for miR-26a/b-5p (pMu_miR-26a/b-5p (295), pMu_miR-26a/b-5p (344)) and for miR-185-5p (pMu_miR-185-5p), induced recovery of the reporter gene expression, while two site mutations for miR-26a/b-5p (pMu_miR-26a/b-5p $(295,344))$ also up-regulated luciferase levels, albeit without the synergistic effect. As expected, there was no difference in the luciferase activity in the mutated miR-203a-3p binding sites (pMu_miR-203a-3p) resulting from the absence of expression of this miRNA in YT cells. 


\begin{tabular}{|c|c|c|c|}
\hline \multicolumn{3}{|c|}{$290 \quad 295$} & \\
\hline Luciferase & $-203 a-3 p$ & $\begin{array}{l}\operatorname{miR}-26 a-5 p \\
\text { miR-26b-5p }\end{array}$ & $\begin{array}{l}\operatorname{miR}-26 a-5 p \\
\text { miR-26b-5p }\end{array}$ \\
\hline \multirow{2}{*}{ MicroRNA } & \multirow{2}{*}{$\underset{\mathrm{kcal} / \mathrm{mol}}{\mathrm{mfe}}$} & \multicolumn{2}{|c|}{ Binding site at 3'UTR KIR3DL3 } \\
\hline & & Wild type & Mutant form \\
\hline $\mathrm{miR}-26 \mathrm{a} / \mathrm{b}-5 \mathrm{p}(295)$ & $-18.7 /-16$ & ${ }^{295}$ ACTTGA $^{300}$ & ${ }^{295} \mathrm{AC} \underline{\mathrm{C}}$ T $\underline{\mathrm{A}} \mathrm{A}^{300}$ \\
\hline $\mathrm{miR}-26 \mathrm{a} / \mathrm{b}-5 \mathrm{p}(344)$ & $-24.0 /-20$ & ${ }^{344}$ ACTTGA $^{349}$ & ${ }^{344} \underline{\text { GCTT }} \underline{\text { AAA }}{ }^{349}$ \\
\hline $\operatorname{miR}-185-5 p$ & -25.2 & ${ }^{312} \mathrm{CTCTCC}^{317}$ & ${ }^{312} \mathrm{CT} \underline{\mathrm{A}} \mathrm{TC}^{317}$ \\
\hline miR-203a-3p & -15.6 & ${ }^{290}$ ATTTCA $^{295}$ & ${ }^{290} \mathrm{~A} \underline{\mathrm{GT}} \underline{\mathrm{A}} \mathrm{CA}^{295}$ \\
\hline
\end{tabular}

(a)

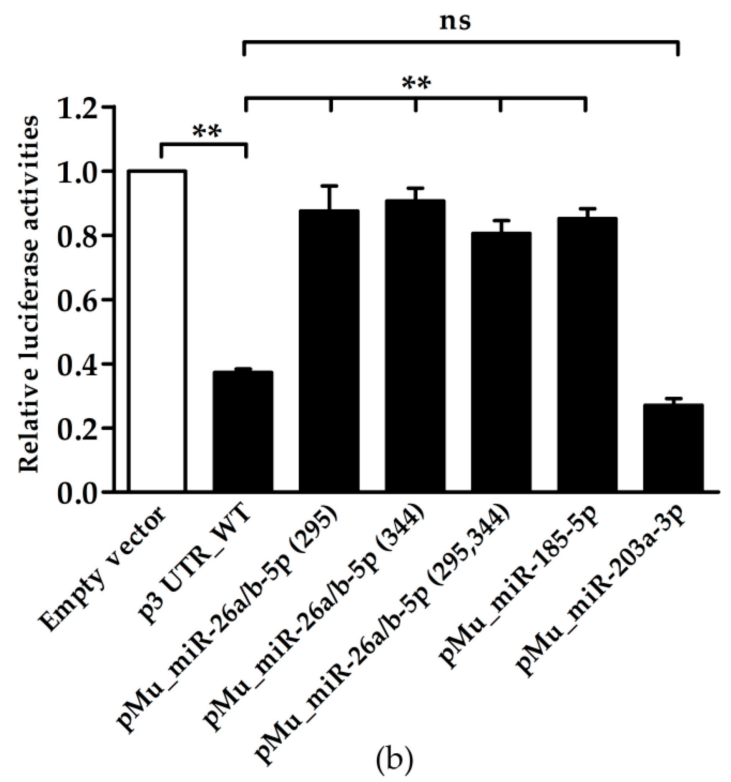

Figure 2. The effect of endogenous miRNAs interacting with the 3'UTR of KIR3DL3 as indicated by luciferase activity. (a) The predicted miRNA binding sites on the 3'UTR of KIR3DL3 are presented in the table. The minimum free energy (mfe) for the binding of each miRNA was calculated by the RNA hybrid program. MiRNA binding sites were mutated by PCR directed mutagenesis and were confirmed by DNA sequencing as shown in the table. The superscript numbers represent the position of the seed sequence binding to the $3^{\prime}$ UTR of KIR3DL3. (b) Results from the dual-luciferase assay in YT cell lines when transfected with luciferase constructs of the 3'UTR wild-type (p3UTR_WT) or mutant forms (mutated miRNA-binding site, pMu_miR). The firefly luciferase activity was normalized by renilla luciferase activity and then relative to empty vector. Data are representative of three independent experiments (mean \pm S.E.M.). ${ }^{* *} p<0.01$ (Student's $t$-test).

\subsection{Knocking Down Candidate miRNAs Resulted in Higher Cell Surface KIR3DL3 Expression}

We determined the influence of candidate miRNAs on the cell surface expression of KIR3DL3. K562, an erythroleukemia cell line with low surface expression of KIR3DL3, was transfected with miRNA inhibitors for miR-26a-5p, miR-26b-5p and miR-185-5p and in combination (miR-26a/b-5p or $\mathrm{miR}-26 \mathrm{a} / \mathrm{b}-5 \mathrm{p} \pm 185-5 \mathrm{p}$ ) leading to elevated KIR3DL3 cell surface expression (Figure 3 ). Compared with the scramble control, endogenous miR-26a-5p, miR-26b-5p, and miR-185-5p were significantly decreased in the miRNA inhibitor transfected K562 cells (Figure 3a). As expected, specifically knocking down miRNAs caused upregulation of the cell surface KIR3DL3 on K562 cells. When compared to negative control (scramble control), inhibiting effect of miR-26a-5p, miR-26b-5p and miR-185-5p significantly increased surface KIR3DL3 expression for both mean fluorescent intensity and percentage of positive cells (Figure 3c,d). In addition, the synergistic effects of miR-26a-5p and miR-26b-5p and 
three miRNA inhibitors (miR-26a/b-5p $\pm 185-5 p$ ) also displayed a strong regulation effect but was not significantly different compared to a single miRNA inhibition (miR-26b-5p and miR-185-5p). However, the regulation efficiency of miR-26a-5p was not as great as miR-26b-5p and miR-185-5p. Consistent with increased protein expression, significantly decreased endogenous mRNA levels were observed in cells after exposure to antisense miRNAs, except for anti-miR-26a-5p (Figure 3b).

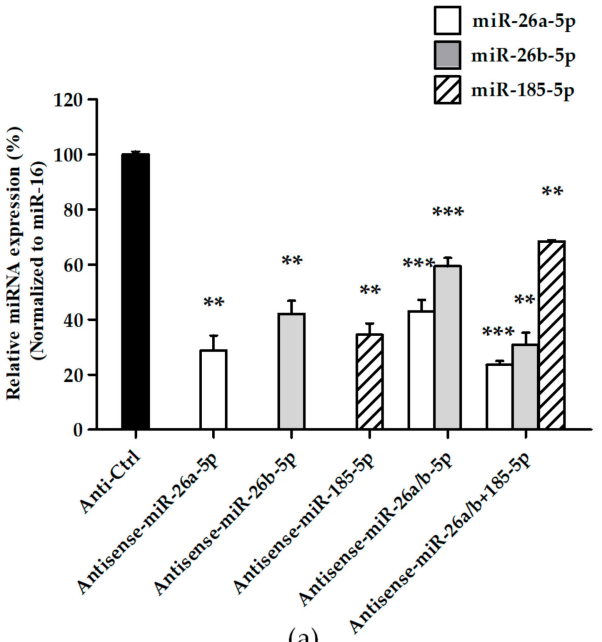

(a)

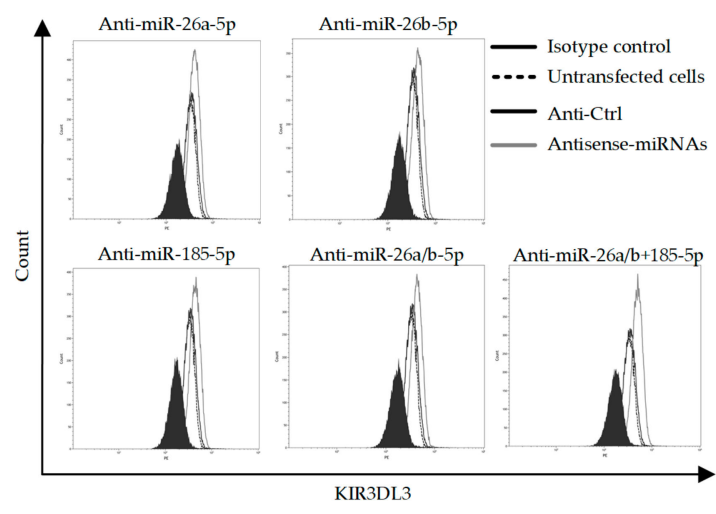

(c)

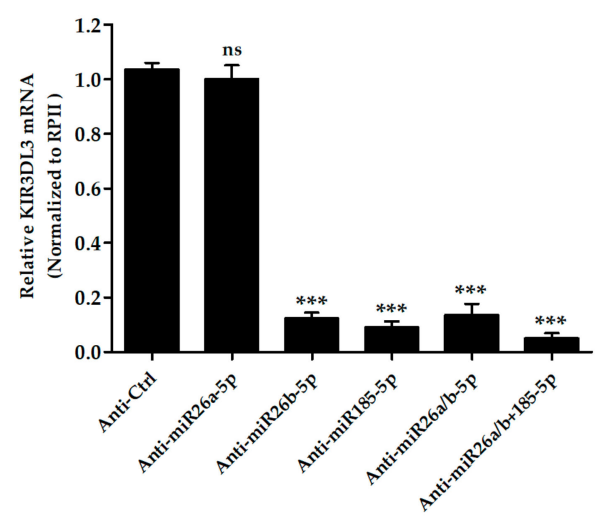

(b)

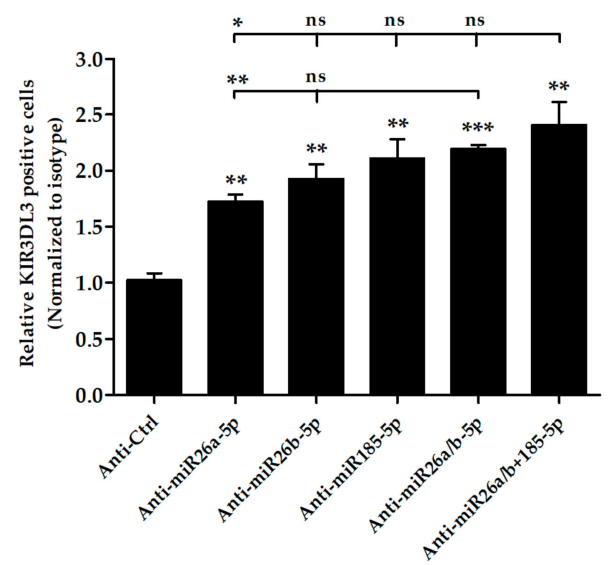

(d)

Figure 3. Knockdown candidate miRNAs increase the expression of KIR3DL3. K562 cells were transfected with $500 \mathrm{nM}$ candidate antisense miRNAs for $48 \mathrm{~h}$ and subsequently assessed for KIR3DL3 expression. (a) Relative expression levels of mature miR-26a-5p, -26b-5p and 185-5p normalized to miR-16 when were compared to the anti-sense control (scramble) are presented. (b) The relative KIR3DL3 mRNA expressions are presented. Total RNA was extracted, and the levels of KIR3DL3 mRNA normalized to RPII were measured by RT-PCR. The figure shows the means \pm S.E.M. from three independent experiments. (c) After transient transfection, cells were examined by flow cytometry for cell surface KIR3DL3 expression. The filled histogram represents cells stained with the isotype antibody. In the open histogram, the dotted line indicates K562 untransfected cells, the solid line shows the data from the negative control (anti-Ctrl or miRNA scramble control) and the gray line represents the transfection of $500 \mathrm{nM}$ antisense miRNAs all stained with the KIR3DL3 antibody. (d) A relative number of positive cells for KIR3DL3 are shown. Results are calculated from three experiments and depicted as mean \pm S.E.M. ${ }^{*}, p<0.05 ;{ }^{* *}, p<0.01 ;{ }^{* * *}, p<0.001$.

\section{Discussion}

It has been unclear how KIR repertoire is created for NK cell clonality. Expression of KIR3DL3 has been proposed to be regulated by DNA methylation [16]. A recent study showed that KIR3DL3 
mRNA was present in purified decidual NK and also peripheral blood NK cells but the corresponding protein was not detected at the cell surface $[14,16]$. Therefore, expression of KIR3DL3 may be inducible in developmental situations or in some diseases. In this study, our data revealed for the first time that KIR3DL3 is also regulated by miRNAs. These results provide a molecular rationale for undetectable KIR3DL3 expression on the cell surface.

Analyses of gene regulation have shown that miRNAs play an important role in controlling many critical cellular processes in NK cells. For example, miR-181 and miR-150 have been found to influence NK cell function, maturation, development, and cell survival [31-33]. Furthermore, the presence of miR-1245 regulates the expression of the NK cell activating receptor, NKG2D [34]. In this study, we used three different target prediction software programs (RNAhybrid, TargetScan, and miRanda) to identify 26 candidate miRNAs that could bind the 3'UTR of KIR3DL3. To refine our analysis, we used previous data from microarray studies that identified a number of miRNAs in NK cells [31] and our own screen of miRNAs in NK cell lines, NK92 and YT, to restrict our validation to three potential miRNAs (miR-26a-5p, -26b-5p and -185-5p) that could modulate surface KIR3DL3 expression. The miRNA miR-34a-5p in the bioinformatic prediction was not included in our analysis as although it has been shown to be up-regulated in immature CD56 $6^{\text {bright }} \mathrm{NK}$ populations and has been proposed to regulate KIR3DL3 expression [18].

Normally, the KIR genotype should be the main determinant of receptor expression on NK cells. Thus, KIR repertoire and NK clonality should be generated by gene regulation. The gene sequence of KIR3DL3 is very similar to those of other KIRs, albeit it is regulated independently [12,16]. Most studies proposed an integrated model for the stochastic expression of KIR by DNA methylation [10,35]. In that, KIR3DL3 displays methylation at the 5' promotor region [16] and exposure to a methyltransferase inhibitor effectively induced KIR3DL3 expression on NK92 cells [16]. In addition to DNA methylation, here, we showed that the knocking down of candidate miRNAs effectively induced KIR3DL3 protein cell surface expression.

High sequence identity is a common feature of the KIR gene family [36] and the 3'UTR is no exception. We analyzed the sequences of the 3'UTR of the KIR genes and found that KIR3DL3 has a high degree of sequence identity with the 3'UTR of other KIRs (86-95\%) as shown in Supplementary Table S3. All three miRNA candidates could bind to 3UTR of all 14 KIR genes (Table S4). These data suggest that modulation of KIRs could be dependent on miRNAs. In addition, it is reasonable to believe that KIR repertoire in different individual clonal NK cells reflects the presence of differential miRNA expression. The diverse KIR receptor patterns consistently correlate with the different stages of NK maturation and their immune responses.

Theoretically, anti-sense of miRNA should not affect mRNA but only miRNA expression. However, in our experiments, we found that the transfection of anti-miRNAs could also reduce the level of KIR3DL3 mRNA (Figure 3b) except anti-miR26a-5p. This is probably due to the kinetic of mRNA and protein turn-over, reducing the mRNA level. This phenomenon has also been observed by others [37]. Interestingly, the observation for anti-miR26a-5p reflects only a direct effect on the expression of this miRNA with a corresponding increase in cell surface expression, albeit, it was the lowest level compared to the others.

K562 cells expressing a low level of endogenous mRNA and protein of KIR3DL3 was used for functional verification and validation by knocking-down the candidate miRNAs. The surface KIR3DL3 was significantly upregulated represented by the percentage of positive cells and mean fluorescent intensity (MFI). Interestingly, there were synergistic effects observed from multiple miRNAs, especially the three-miR combination. Moreover, different miRNAs differed in the degree they modulated expression of KIR3DL3. Unfortunately, the analysis of gain of function approach by transfection with the mimic candidate miRNAs was not possible because the over-expression of miRNA candidates led to cell death (data not shown). It was recently observed that over-expression of miR-185-5p induced apoptosis by directly targeting on anti-apoptotic genes, B-cell lymphoma (BCL)2 and BCL2L1, in prostate cancer cell lines [38] as well as the effect of miR-26a-5p [39]. In addition, miR-26a/b is a potential 
autophagy inhibitor in hepatocellular carcinoma (HCC) to inhibit the expression of serine/threonine protein kinase ULK1 promoting cell apoptosis [40]. Therefore, overexpression of miR-26a-5p, -26b-5p and -185-5p might cause toxicity to cells. These observations were suggestive by most putative gene targets exclusively intermediated in apoptotic pathways by these candidate miRNAs (Table S5).

A limitation of this study was that in our hands the NK92 cell line was resistant to transfection and, therefore, the YT cell line was used to evaluate the miRNA-mRNA interactions by the dual-luciferase reporter assay. However, initial data analysis suggested that the 3'UTR of KIR3DL3 was a potential target for three miRNAs (miR-26a-5p, -26b-5p and -185-5p) expressed in YT cells (Figure S1).

In summary, we have identified for the first time a miRNA regulatory mechanism that modulates KIR3DL3 surface expression. This mechanism could contribute to and maintain clonal expression patterns of KIRs on immune cells relevant to immune responses. The information could assist in further studying the function and ligand identification of KIR3DL3.

Supplementary Materials: The following are available online at http://www.mdpi.com/2073-4425/10/8/603/s1, Table S1: Primer sets for KIR3DL3 and miRNAs detections, Table S2: Killer Immunoglobulin-like receptor (KIR) expression profiles in the NK-92 cell line, Table S3: The percent sequence identity between the 3' untranslated region (3'UTR) of different KIRs. Table S4: Computational prediction of three candidate miRNAs binding on 3'UTR of 14 KIR genes, Table S5: Target genes for miR-26a-5p, -26b-5p and -185-5p, Figure S1: Endogenous miRNA expression levels in the YT and K562 cell lines. Relative candidate miRNA expressions in YT and K562 are presented. The expression of miR-203a-3p was not detected in YT but very small amount was present in K562.

Author Contributions: Designed and planned the study, R.N., A.J. and C.L.; Executed and analyzed the experiments, R.N.; Critically discussed the data. R.N, A.J., C.L., and S.G.; Wrote the first draft of the manuscript, S.G., A.J. and C.L.; Finalized the manuscript, R.N., C.L., and S.G.; all authors reviewed and approved the manuscript.

Funding: This research was funded by the Royal Golden Jubilee program (RGJ) and The Centre for Research and Development of Medical Diagnostic Laboratories and R.N. was funded by the Royal Golden Jubilee program (RGJ) grant number [RGJ:PHD/0040/2556], Thailand Research Fund (TRF) and the APC was funded by The University of Western Australia.

Acknowledgments: We thank Ashley Moffett for providing YT-KIR3DL3 and primary mouse anti-KIR3DL3 antibody. We thank our colleagues at the Institute for Immunology and Infectious Diseases, Murdoch University, Australia. This work was supported by the Centre for Research and Development of Medical Diagnostic Laboratories (CMDL) and RGJ. This work was also, in part, supported by a National Health and Medical Research Council grant to S.G. (APP1148284).

Conflicts of Interest: The authors declare no conflict of interest. The funding sponsors had no role in the design of the study, in the analyses, or the interpretation of data; in the writing of the manuscript, and in the decision to publish the results.

\section{References}

1. Vilches, C.; Parham, P. KIR: diverse, rapidly evolving receptors of innate and adaptive immunity. Annu. Rev. Immunol. 2002, 20, 217-251. [CrossRef] [PubMed]

2. Yang, J.H.; Chen, M.J.; Chen, H.F.; Lee, T.H.; Ho, H.N.; Yang, Y.S. Decreased expression of killer cell inhibitory receptors on natural killer cells in eutopic endometrium in women with adenomyosis. Hum. Reprod. 2004, 19, 1974-1978. [CrossRef] [PubMed]

3. Ljunggren, H.G.; Karre, K. In search of the 'missing self': MHC molecules and NK cell recognition. Immunol. Today 1990, 11, 237-244. [CrossRef]

4. Valiante, N.M.; Uhrberg, M.; Shilling, H.G.; Lienert-Weidenbach, K.; Arnett, K.L.; D'Andrea, A.; Phillips, J.H.; Lanier, L.L.; Parham, P. Functionally and structurally distinct NK cell receptor repertoires in the peripheral blood of two human donors. Immunity 1997, 7, 739-751. [CrossRef]

5. Husain, Z.; Alper, C.A.; Yunis, E.J.; Dubey, D.P. Complex expression of natural killer receptor genes in single natural killer cells. Immunology 2002, 106, 373-380. [CrossRef] [PubMed]

6. Hsu, K.C.; Chida, S.; Geraghty, D.E.; Dupont, B. The killer cell immunoglobulin-like receptor (KIR) genomic region: gene-order, haplotypes and allelic polymorphism. Immunol. Rev. 2002, 190, 40-52. [CrossRef] [PubMed] 
7. Wilson, M.J.; Torkar, M.; Haude, A.; Milne, S.; Jones, T.; Sheer, D.; Beck, S.; Trowsdale, J. Plasticity in the organization and sequences of human KIR/ILT gene families. Proc. Natl. Acad. Sci. USA 2000, 97, 4778-4783. [CrossRef] [PubMed]

8. Rajagopalan, S.; Long, E.O. A human histocompatibility leukocyte antigen (HLA)-G-specific receptor expressed on all natural killer cells. J. Exp. Med. 1999, 189, 1093-1100. [CrossRef]

9. Chan, H.W.; Miller, J.S.; Moore, M.B.; Lutz, C.T. Epigenetic control of highly homologous killer Ig-like receptor gene alleles. J. Immunol. 2005, 175, 5966-5974. [CrossRef]

10. Santourlidis, S.; Trompeter, H.I.; Weinhold, S.; Eisermann, B.; Meyer, K.L.; Wernet, P.; Uhrberg, M. Crucial role of DNA methylation in determination of clonally distributed killer cell Ig-like receptor expression patterns in NK cells. J. Immunol. 2002, 169, 4253-4261. [CrossRef]

11. Uhrberg, M. Shaping the human NK cell repertoire: an epigenetic glance at KIR gene regulation. Mol. Immunol. 2005, 42, 471-475. [CrossRef] [PubMed]

12. Torkar, M.; Norgate, Z.; Colonna, M.; Trowsdale, J.; Wilson, M.J. Isotypic variation of novel immunoglobulinlike transcript/killer cell inhibitory receptor loci in the leukocyte receptor complex. Eur. J. Immunol. 1998, 28, 3959-3967. [CrossRef]

13. Vilches, C.; Rajalingam, R.; Uhrberg, M.; Gardiner, C.M.; Young, N.T.; Parham, P. KIR2DL5, a novel killer-cell receptor with a D0-D2 configuration of Ig-like domains. J. Immunol. 2000, 164, 5797-5804. [CrossRef] [PubMed]

14. Long, E.O.; Barber, D.F.; Burshtyn, D.N.; Faure, M.; Peterson, M.; Rajagopalan, S.; Renard, V.; Sandusky, M.; Stebbins, C.C.; Wagtmann, N.; et al. Inhibition of natural killer cell activation signals by killer cell immunoglobulin-like receptors (CD158). Immunol. Rev. 2001, 181, 223-233. [CrossRef] [PubMed]

15. Leaton, L.A.; Shortt, J.; Kichula, K.M.; Tao, S.; Nemat-Gorgani, N.; Mentzer, A.J.; Oppenheimer, S.J.; Deng, Z.; Hollenbach, J.A.; Gignoux, C.R.; et al. Conservation, Extensive Heterozygosity, and Convergence of Signaling Potential All Indicate a Critical Role for KIR3DL3 in Higher Primates. Front. Immunol. 2019, 10, 24. [CrossRef] [PubMed]

16. Trundley, A.E.; Hiby, S.E.; Chang, C.; Sharkey, A.M.; Santourlidis, S.; Uhrberg, M.; Trowsdale, J.; Moffett, A. Molecular characterization of KIR3DL3. Immunogenetics 2006, 57, 904-916. [CrossRef] [PubMed]

17. Trompeter, H.I.; Gomez-Lozano, N.; Santourlidis, S.; Eisermann, B.; Wernet, P.; Vilches, C.; Uhrberg, M. Three structurally and functionally divergent kinds of promoters regulate expression of clonally distributed killer cell Ig-like receptors (KIR), of KIR2DL4, and of KIR3DL3. J. Immunol. 2005, 174, 4135-4143. [CrossRef] [PubMed]

18. Pesce, S.; Squillario, M.; Greppi, M.; Loiacono, F.; Moretta, L.; Moretta, A.; Sivori, S.; Castagnola, P.; Barla, A.; Candiani, S.; et al. New miRNA Signature Heralds Human NK Cell Subsets at Different Maturation Steps: Involvement of miR-146a-5p in the Regulation of KIR Expression. Front. Immunol. 2018, 9, 2360. [CrossRef]

19. Beaulieu, A.M.; Bezman, N.A.; Lee, J.E.; Matloubian, M.; Sun, J.C.; Lanier, L.L. MicroRNA function in NK-cell biology. Immunol. Rev. 2013, 253, 40-52. [CrossRef]

20. Ni, F.; Guo, C.; Sun, R.; Fu, B.; Yang, Y.; Wu, L.; Ren, S.; Tian, Z.; Wei, H. MicroRNA transcriptomes of distinct human NK cell populations identify miR-362-5p as an essential regulator of NK cell function. Sci. Rep. 2015, 5, 9993. [CrossRef]

21. Ambros, V. The functions of animal microRNAs. Nature 2004, 431, 350-355. [CrossRef] [PubMed]

22. Baek, D.; Villen, J.; Shin, C.; Camargo, F.D.; Gygi, S.P.; Bartel, D.P. The impact of microRNAs on protein output. Nature 2008, 455, 64-71. [CrossRef] [PubMed]

23. Kozomara, A.; Griffiths-Jones, S. miRBase: annotating high confidence microRNAs using deep sequencing data. Nucleic Acids Res. 2014, 42, D68-D73. [CrossRef] [PubMed]

24. Kruger, J.; Rehmsmeier, M. RNAhybrid: microRNA target prediction easy, fast and flexible. Nucleic Acids Res. 2006, 34, W451-W454. [CrossRef] [PubMed]

25. Bartel, D.P. Targetscanhuman: Prediction of microRNA Targets. Available online: http://www.targetscan.org/ vert_61/ (accessed on 30 June 2019).

26. Betel, D. Miranda: A Comprehensive Resource of microRNA Target Predictions and Expression Profiles. Available online: http://www.microrna.org/microrna/getDownloads.do (accessed on 30 June 2019).

27. Chen, C.; Ridzon, D.A.; Broomer, A.J.; Zhou, Z.; Lee, D.H.; Nguyen, J.T.; Barbisin, M.; Xu, N.L.; Mahuvakar, V.R.; Andersen, M.R.; et al. Real-time quantification of microRNAs by stem-loop RT-PCR. Nucleic Acids Res. 2005, 33, e179. [CrossRef] [PubMed] 
28. Varkonyi-Gasic, E.; Wu, R.; Wood, M.; Walton, E.F.; Hellens, R.P. Protocol: a highly sensitive RT-PCR method for detection and quantification of microRNAs. Plant Methods 2007, 3, 12. [CrossRef]

29. Tajik, N.; Shahsavar, F.; Nasiri, M.; Radjabzadeh, M.F. Compound KIR-HLA genotype analyses in the Iranian population by a novel PCR-SSP assay. Int. J. Immunogenet. 2010, 37, 159-168. [CrossRef] [PubMed]

30. Stern-Ginossar, N.; Gur, C.; Biton, M.; Horwitz, E.; Elboim, M.; Stanietsky, N.; Mandelboim, M.; Mandelboim, O. Human microRNAs regulate stress-induced immune responses mediated by the receptor NKG2D. Nat. Immunol. 2008, 9, 1065-1073. [CrossRef]

31. Bezman, N.A.; Cedars, E.; Steiner, D.F.; Blelloch, R.; Hesslein, D.G.; Lanier, L.L. Distinct requirements of microRNAs in NK cell activation, survival, and function. J. Immunol. 2010, 185, 3835-3846. [CrossRef]

32. Bezman, N.A.; Chakraborty, T.; Bender, T.; Lanier, L.L. miR-150 regulates the development of NK and iNKT cells. J. Exp. Med. 2011, 208, 2717-2731. [CrossRef]

33. Cichocki, F.; Felices, M.; McCullar, V.; Presnell, S.R.; Al-Attar, A.; Lutz, C.T.; Miller, J.S. Cutting edge: microRNA-181 promotes human NK cell development by regulating Notch signaling. J. Immunol. 2011, 187, 6171-6175. [CrossRef] [PubMed]

34. Espinoza, J.L.; Takami, A.; Yoshioka, K.; Nakata, K.; Sato, T.; Kasahara, Y.; Nakao, S. Human microRNA-1245 down-regulates the NKG2D receptor in natural killer cells and impairs NKG2D-mediated functions. Haematologica 2012, 97, 1295-1303. [CrossRef] [PubMed]

35. Chan, H.W.; Kurago, Z.B.; Stewart, C.A.; Wilson, M.J.; Martin, M.P.; Mace, B.E.; Carrington, M.; Trowsdale, J.; Lutz, C.T. DNA methylation maintains allele-specific KIR gene expression in human natural killer cells. J. Exp. Med. 2003, 197, 245-255. [CrossRef] [PubMed]

36. Kelley, J.; Walter, L.; Trowsdale, J. Comparative genomics of natural killer cell receptor gene clusters. PLoS Genet. 2005, 1, 129-139. [CrossRef] [PubMed]

37. Liu, B.; Wu, X.; Liu, B.; Wang, C.; Liu, Y.; Zhou, Q.; Xu, K. MiR-26a enhances metastasis potential of lung cancer cells via AKT pathway by targeting PTEN. Biochim. Biophys. Acta. 2012, 1822, 1692-1704. [CrossRef] [PubMed]

38. Ostadrahimi, S.; Abedi Valugerdi, M.; Hassan, M.; Haddad, G.; Fayaz, S.; Parvizhamidi, M.; Mahdian, R.; Fard Esfahani, P. miR-1266-5p and miR-185-5p Promote Cell Apoptosis in Human Prostate Cancer Cell Lines. Asian. Pac. J. Cancer Prev. 2018, 19, 2305-2311.

39. Rizzo, M.; Berti, G.; Russo, F.; Fazio, S.; Evangelista, M.; D'Aurizio, R.; Pellegrini, M.; Rainaldi, G. Discovering the miR-26a-5p Targetome in Prostate Cancer Cells. J. Cancer 2017, 8, 2729-2739. [CrossRef] [PubMed]

40. Jin, F.; Wang, Y.; Li, M.; Zhu, Y.; Liang, H.; Wang, C.; Wang, F.; Zhang, C.Y.; Zen, K.; Li, L. MiR-26 enhances chemosensitivity and promotes apoptosis of hepatocellular carcinoma cells through inhibiting autophagy. Cell Death Dis. 2017, 8, e2540. [CrossRef] 